\title{
107.1-GBPS NET-RATE TRANSMISSION OVER A JOINT 51km-FIBER-AND-10.7m-WIRELESS LINK FOR TERAHERTZ RADIO ACCESS NETWORKS
}

\author{
Aleksejs Udalcovs ${ }^{*}$, Shi Jia ${ }^{2}$, Lu Zhang ${ }^{3,4}$, Oskars Ozolins ${ }^{1,3}$, Xiaodan Pang ${ }^{3}$, Deming
} Kong $^{2}$, Xianbin Yu ${ }^{5}$, Shilin Xiao ${ }^{4}$, Sergei Popov ${ }^{3}$, Jiajia Chen ${ }^{3,6}$, Toshio Morioka ${ }^{2}$, Hao $\mathrm{Hu}^{2}$, and Leif K. Oxenløwe ${ }^{2 *}$

\author{
${ }^{I}$ RISE Research Institutes of Sweden AB, Isafjordsgatan 22, 16440 Kista, Sweden \\ ${ }^{2}$ DTU Fotonik, Technical University of Denmark, Ørsteds Plads 343, 2800 Kgs. Lyngby, Denmark \\ ${ }^{3}$ Schools of EECS and SCI, KTH Royal Institute of Technology, Isafjordsgatan 22, 16440 Kista, Sweden \\ ${ }^{4}$ Laboratory of Advanced Opt. Comm. Syst. and Netw., Shanghai Jiao Tong University, 200240 Shanghai, China \\ ${ }^{5}$ College of Information Science and EE, Zhejiang University, 310027 Hangzhou, China \\ ${ }^{6}$ Chalmers University of Technology, Chalmersplatsen 4, 41296 Gothenburg, Sweden \\ *aleksejs.udalcovs@ri.se,lkox@fotonik.dtu.dk
}

Keywords: THZ-COMMUNICATIONS, PHOTONIC RADIO, FIBER-WIRELESS LINK, RADIO ACCESS NETWORKS, BEYOND 5G

\begin{abstract}
107.1- and 93.9-Gbps net-rate (gross-rate 133.9- and 101.3-Gbps) OFDM transmission on a 408-GHz carrier frequency is experimentally demonstrated over a joint fiber-wireless link, 51-km single-mode fiber and $10.7-\mathrm{m}$ free-space, with bit-errorrates below the $20 \%$ soft- and $7 \%$ hard-decision FEC thresholds of $2.7 \times 10^{-2}$ and $3.8 \times 10^{-3}$, respectively.
\end{abstract}

\section{Introduction}

While mobile communications move towards the fifth generation $(5 \mathrm{G})$, it has become obvious that networks beyond 5G (B5G) will have to tackle fundamental performance limitations related to the available bandwidth in the wireless spectrum, transmission and processing delay, cost and energy consumption [1]. Considering that the currently allocated frequency spectrum for $5 \mathrm{G}$ has almost reached its limits, the terahertz-wave band $(0.1-10 \mathrm{THz})$ is gaining more attention for the upcoming B5G [2]. As a result, research on $\mathrm{THz}$ and sub-THz radio access networks is of great importance to utilize the unlicensed bandwidth in higher wireless frequency bands [3]. First, the $\mathrm{THz}$ wireless technology has a potential of delivering fiber-optic speeds "out of the fiber" offering terabits per second (Tbps) wireless connectivity for base-band units (BBUs) or even for radio remote units (RRUs). Second, $\mathrm{THz}$ communications are expected to be used for wireless access and backhaul networking to ensure high-speed Internet access everywhere, including areas where an optical fiber cannot be deployed due to geographical and infrastructure operator cost issues [4]. Moreover, such areas will expand due to the increasing number of RRUs per base-station. Hence, hundreds of gigabits per second will be required in transmission to or between BBU pools or between BBU pool and RRUs.

The $\mathrm{THz}$ channel itself is important to explore and develop, and several demonstrations have been reported in the $300 \mathrm{GHz}$ range, e.g., [5], and in the $400 \mathrm{GHz}$ range, e.g., [6], [7], focusing on capacity and wireless reach. However, if the application scenario is related to the reach extension in access networks, apart from the targeted high data rates and long wireless transmission, an overall reach for the joint fiber and wireless link is another critical parameter. Such joint fiberwireless links should consist of at least two segments: $(i)$ an optical fiber link, where two optical wavelengths (one carries data, the other is for $\mathrm{THz}$ generation) separated by the desired $\mathrm{THz}$ carrier frequency are transmitted; and (ii) a free-space segment for $\mathrm{THz}$ wireless transmission. Both these segments should be long enough, but they share an optical budget and contributes to an overall signal-to-noise ratio (SNR), which impacts a transmission performance. Seamless integration of radio and optical networks in a physical layer reduces network complexity and ensures high capacity in the upper transport and application layers, by reducing the need for additional signaling and management, especially considering a small-cell configuration [4]. In spite of that, only a few demonstrations report considerably high net-rates for joint fiber-and-THzwireless links: in [8], a net-rate of $59 \mathrm{Gbps}$ is demonstrated for a link that consists of short fiber span (the length is not mentioned) and $5 \mathrm{~cm}$ short wireless transmission at a carrier frequency of $325 \mathrm{GHz}$; and recently, in [9], a gross-rate of 132 Gbps with bit-error-rate (BER) under $4 \times 10^{-2}$ is experimentally demonstrated for a single carrier signal at $450 \mathrm{GHz}$ after transmission over a $20 \mathrm{~km}$ fiber and $1.8 \mathrm{~m}$ wireless distance. However, both solutions have very limited applications due to a relatively low distance and/or signal quality performance. In this context, we modify our THz-wireless link presented in [6] by substituting a THz-wave generation setup and by adding a $51 \mathrm{~km}$ long single-mode fiber (SMF) span to construct a joint fiber-wireless link operating with carrier frequencies in the $>400 \mathrm{GHz}$ band and wireless reach above $10 \mathrm{~m}$. The single carrier at $408 \mathrm{GHz}$ was generated by an optical frequency comb generator (OFCG) using a so-called photonic radio setup, and we use orthogonal frequency division multiplexing 
(OFDM) for data transmission over the joint link. We achieved a BER below the $20 \%$ soft-decision forward error correction (SD-FEC) threshold of $2.7 \times 10^{-2}$ [10] for a gross-rate of 133.9 Gbps using 16-level quadrature amplitude modulation (16QAM)-modulated subcarriers of the OFDM signals, yielding a transmitted net-rate of $107.1 \mathrm{Gbps}$. The $7 \%$ harddecision FEC (HD-FEC) threshold of $3.8 \times 10^{-3}$ is achieved for a gross-rate of $101.3 \mathrm{Gbps}$ using quadrature phase-shift keying (QPSK)-modulated OFDM subcarriers, leading to a net-rate of 93.9 Gbps. Furthermore, we highlight that the BER requirement of $4 \times 10^{-2}$ for $27 \%$ SD-FEC [9] is met for a grossrate of $159.6 \mathrm{Gbps}$; it brings a slightly higher net-rate of 116.5 Gbps, but at a cost of complexity that may be too high for optical and radio networks beyond 5G. In any case, these results, to the best of our knowledge, demonstrate for the first time the joint fiber-wireless link with net-rates above $100 \mathrm{Gbps}$ after more than $50 \mathrm{~km}$ long fiber and $10 \mathrm{~m}$ long THz-wireless transmission using a carrier frequency above $400 \mathrm{GHz}$, which overwhelms the previous demonstrations.

\section{Experimental Setup}

The experimental setup is shown in Fig. 1. First, we generate two optical tones using an optical frequency comb generator based on an external cavity laser (ECL) and phase modulator that are driven by a $24 \mathrm{GHz}$ radio frequency (RF) signal from an electrical local oscillator (LO). The generated comb signal is amplified by an Erbium doped fiber amplifier (EDFA) before two tones spaced by $408 \mathrm{GHz}$ are filtered and separated by a wavelength selective switch (WSS).
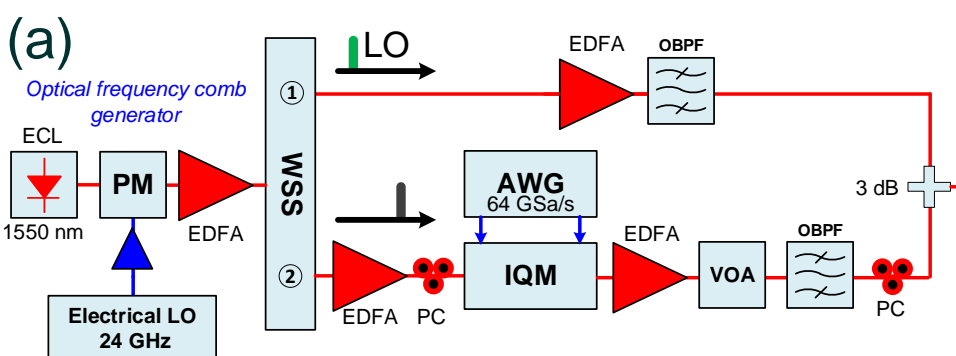

$34 \mathrm{~km}+17 \mathrm{~km}$
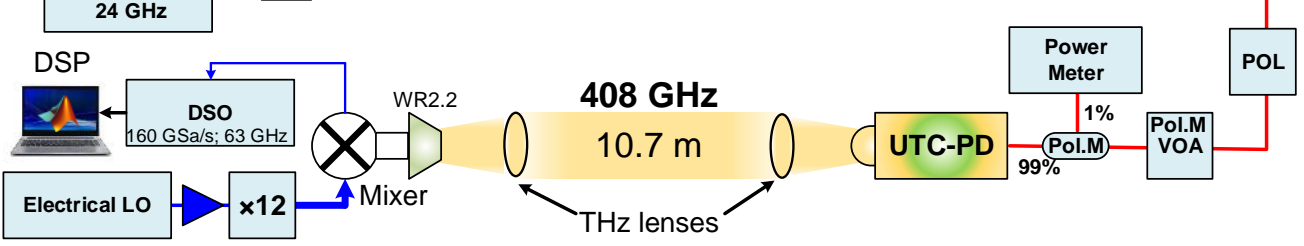

(b)

Fig. 1 (a) Experimental setup of the fiber-THz-wireless link used for the simultaneous transmission of the OFDMmodulated data; and (b) the moment from the experiment. Acronyms: ECL - external cavity laser; PM - phase modulator;

EDFA - Erbium-doped fiber amplifier; LO - local oscillator; WSS - wavelength selective switch; PC - polarization controller; AWG - arbitrary waveform generator; IQM - In-phase and quadrature modulator; VOA - variable optical attenuator; OBPF - optical bandpass filter; POL - polarizer; Pol. M. - polarization maintaining; UTC-PD - uni-travelling carrier photodiode; DSO - digital storage oscilloscope; DSP - digital signal processing

Second, one tone (port 1) is used as an optical LO signal for heterodyne mixing to generate the $\mathrm{THz}$ carrier but is first amplified by an EDFA and filtered by an optical bandpass filter (OBPF) to get rid of excessive noise from the amplifier. The other tone (port 2) is used for carrying data. This tone is amplified, aligned in polarization (polarization controller, PC), and launched to an in-phase and quadrature modulator (IQM). A two-channel arbitrary waveform generator (AWG, 64 $\mathrm{GSa} / \mathrm{s}$ ) is used to generate an IQ-QFDM signal having the length of the inverse fast Fourier transformation (IFFT) and a cyclic prefix (CP) set to 1024 and 16, respectively. A MATLAB generated random binary sequence is used to generate the OFDM symbols. The modulated QPSK/16QAM-OFDM optical signals are amplified, their power levels are adjusted by the variable optical attenuator (VOA) to keep the best power ratio for the highest photo-mixing efficiency in the uni-travelling carrier photodiode (UTC-PD) [11], then the out-of-band noise is filtered by an OBPF, and, finally, the polarization state is controlled by a PC.

Third, after an optical combiner, the optical LO and the IQOFDM-modulated signals are jointly transmitted over two single-mode fiber (SMF) spans - a $34 \mathrm{~km}$ super-large area
(SLA) fiber and a $17 \mathrm{~km}$ inverse dispersion fiber (IDF). These two spans were dispersion matched. After the fiber transmission, the optical signals are amplified, filtered, and their polarizations adjusted. In addition, a polarization maintaining (Pol. M.) VOA is used to control the optical power launched into the UTC-PD for $\mathrm{THz}$ wave generation and wireless transmission. The UTC-PD generates the $\mathrm{THz}$ wave at a $408 \mathrm{GHz}$ carrier frequency and emits it into a $10.7 \mathrm{~m}$ lineof-sight wireless link, where a pair of THz lenses (having 100 $\mathrm{mm}$ diameter and $200 \mathrm{~mm}$ focus length) are used to collimate the $\mathrm{THz}$ beam.

Fourth, at the receiver, the $\mathrm{THz}$ signal is down-converted to an intermediate frequency by a sub-harmonic Schottky mixer driven by a 12-times multiplied RF signal from an electrical LO. The IF signal at radio frequency $30 \mathrm{GHz}$ is amplified, detected and then converted to digital samples by a real-time digital storage oscilloscope (DSO, $160 \mathrm{GSa} / \mathrm{s}$ and $63 \mathrm{GHz}$ ) for digital signal processing (DSP). Finally, we use a typical DSP stack for the OFDM signals. Besides the linear equalization (LE), phase noise compensation (PNC) and nonlinear equalization (NLE) are also used for channel equalization. The LE is used to compensate for the linear response and reduce 
the additive noise influence of the system. Subsequently, the PNC is implemented using a least-squares method. Finally, a simplified Volterra series nonlinear model, which considers the 2 nd-order and the 3rd-order distortion terms, is used for mitigating the nonlinearity impairment.

\section{Results and Discussion}

After the fiber-THz-wireless transmission, we evaluate the single-channel QPSK-/16QAM-OFDM signal. First, we analyze how the channel pre-FEC bitrate (gross-rate) changes with pilot symbol overhead (see Fig. 2 (a)). The results here are after the nonlinear channel equalization (Volterra filtering) and phase compensation. A pilot symbol overhead of $10 \%$ means that every symbol per 10 OFDM symbols are followed by a pilot symbol. Figure 2 (a) also shows how the BER changes with the pilot symbol overhead. For the considered channel and the first OFDM configuration (1024 IFFT points, 900 QPSK-modulated subcarriers), we find that a pilot symbol overhead $(\mathrm{OH})$ of $10 \%$ achieves a BER below $3.8 \times 10^{-3}$. This corresponds to a pre-FEC gross-rate of $101 \mathrm{Gbps}$ (a net-rate 93.9 Gbps). The second OFDM configuration (1024 IFFT points, 560 16QAM-modulated subcarriers) achieves a BER below $2.7 \times 10^{-2}$, which yields a net-rate of 100.8 Gbps. However, these results are obtained for the highest optical power to the UTC-PD, which may lead to saturation. Therefore, optical power sweep is required to check the BER vs. optical power performance for a lower $\mathrm{OH}$ and for a larger number of OFDM subcarriers. Consequently, Fig. 2 (b) presents the BER versus optical power to the UTC-PD for four selected cases that fulfills a number of BER threshold set by a different type of FEC: (i) 101.3 Gbps gross-rate, which corresponds to a pilot symbol overhead of $10 \%$ and fulfills the HD-FEC requirement $\left(3.8 \times 10^{-3}\right)$; (ii) $126 \mathrm{Gbps}$ gross-rate and $10 \% \mathrm{OH}$; (iii) $133.9 \mathrm{Gbps}$ gross-rate, which corresponds to a $4 \% \mathrm{OH}$ and fulfills the $20 \%$ SD-FEC requirement $\left(2.7 \times 10^{-2}\right)$; and (iv) 159.6 Gbps gross-rate, which corresponds a OFDM configuration with 684 16QAM-subcarriers and a 7\% $\mathrm{OH}$ and fulfills the $27 \%$ SD-FEC requirement $\left(4 \times 10^{-2}\right)$.
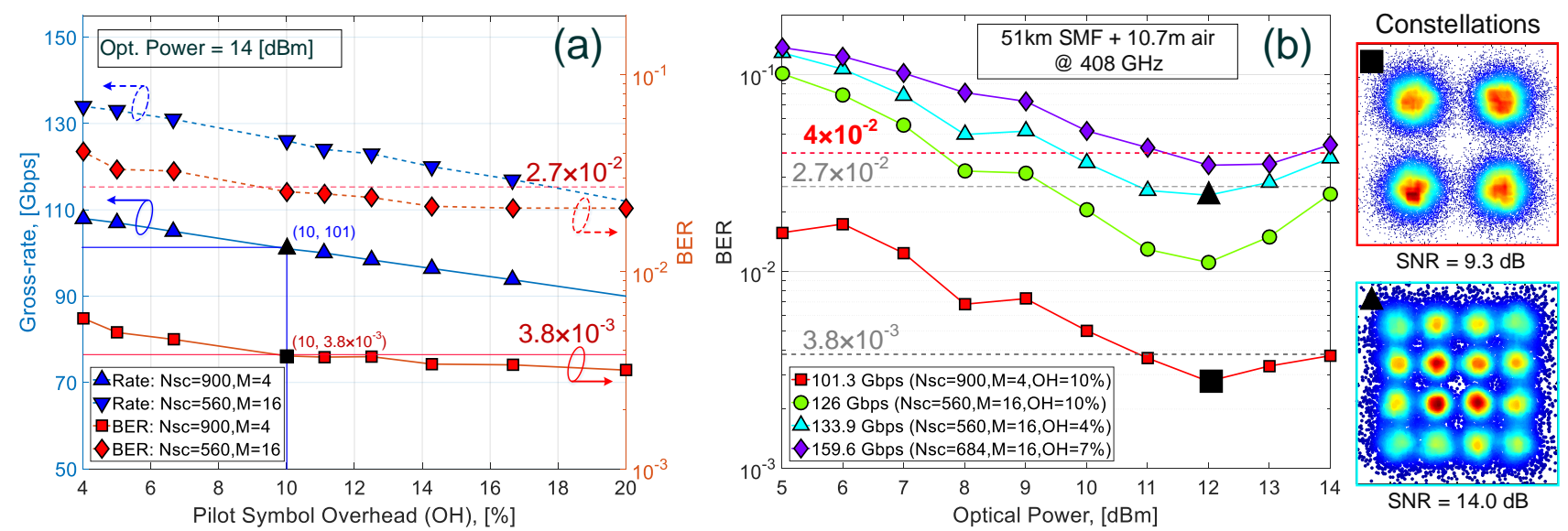

Fig. 2 Experimental results showing (a) the OFDM channel pre-FEC gross-rate) and corresponding BER vs. the pilot symbol overhead for the $14 \mathrm{dBm}$ optical power launched into the UTC-PD; and (b) BER vs. the optical power launched into the UTC-PD for four cases: $101.3 \mathrm{Gbps}, 126 \mathrm{Gbps}, 133.9 \mathrm{Gbps}$, and $159.6 \mathrm{Gbps}$ gross-rates

The lowest BERs are observed for the $+12 \mathrm{dBm}$ optical power, and the highest average signal-to-noise-ratio (SNR) of (i) 9.3 $\mathrm{dB}$, (ii) $14.5 \mathrm{~dB}$, (iii) $14.0 \mathrm{~dB}$, and (iv) $13.2 \mathrm{~dB}$ are achieved for the considered cases. Such low SNR is not only due to the performance of the $\mathrm{THz}$ components, such as the Schottky mixer and the UTC-PD with its poor conversion efficiency (0.15 A/W), but also due to fiber transmission loss. The constellation diagrams for the most significant cases are shown as insets in Fig. 2 (b) and stamp the transmission performance of the joint fiber-and-THz-wireless link.

\section{Concluding Remarks}

We have demonstrated the joint $\mathrm{THz}$ fiber-wireless link in the $>400 \mathrm{GHz}$ carrier frequency range by transmitting a single channel $408 \mathrm{GHz}$ carrier with $93.9 \mathrm{Gbps}$ and $107.1 \mathrm{Gbps}$ (gross-rates: $101.3 \mathrm{Gbps}$ and $133.9 \mathrm{bps}$ ) QPSK-/16QAMOFDM over $51 \mathrm{~km}$ SMF and $10.7 \mathrm{~m}$ free space with BER below the $7 \%$ HD-FEC threshold of $3.8 \times 10^{-3}$ and below the $20 \%$ SD-FEC threshold of $2.7 \times 10^{-2}$. The $\mathrm{THz}$ carrier at 408 $\mathrm{GHz}$ was generated via photonic heterodyning of two tones from an optical frequency comb generator based on phase modulator. The presented results pave the way towards the delivering of fiber-optic speeds "out of the fiber", while extending the radio access networking for future B5G communications.

\section{Acknowledgement}

This work was supported by the EU H2020-MSCA projects COFUNDfellowsDTU (gr. no. 713683), FiWiN5G (gr. no. 642355), and NEWMAN (gr. no. 752826), CoE SPOC (DNRF123), Villum grant 2MAC, China Postdoctoral Science Foundation (gr. no. 2017M611990), Swedish Research Council (VR) within the PHASE project (2016-04510), Swedish Foundation for Strategic Research (SSF), Göran Gustafsson Foundation, National Natural Science Foundation of China (gr. no. 61331010, gr. no. 61722108, gr. no. 61771424, gr. no. 61775137 , and gr. no. 61671212 ), and by VINNOVA within the Utvecklingscentrum för mjukvarustyrda optiska accessnät (no. 2017-01559). 


\section{References}

[1] A.-A. A. Boulogeorgos, A. Alexiou, T. Merkle, et al.: 'Terahertz Technologies to Deliver optical Network Quality of Experience in Wireless Systems Beyond 5G', IEEE Communications Magazine, 2018, 56, (6), 141-151, DOI: 10.1109/MCOM.2018.1700890

[2] K. M. Saidul Huq, J. M. Jornet, W. H. Gerstacker, et al.: ' $\mathrm{THz}$ Communications for Mobile Heterogeneous Networks', IEEE Communications Magazine, 2018, 56, (6), pp. 94-95, DOI: 10.1109/MCOM.2018.8387209

[3] T. Nagatsuma, 'THz Communication Systems', in Proc. OFC, Los Angeles, USA, March 2017, tutorial Tu3B.1.

[4] A. Kanno, P. T. Dat, N. Sekine, et al.: 'Seamless FiberWireless Bridge in the Millimeter and Terahertz-Wave Bands', Journal of Lightwave Technology, 2016, 34, (20), pp. 4794-4801, DOI: $10.1109 /$ JLT.2016.2543240

[5] T. Harter, C. Fullner, J. N. Kemal, et al.: '110-m THz Wireless Transmission at $100 \mathrm{Gbit} / \mathrm{s}$ Using a KramersKronig Schottky Barrier Diode Receiver', in Proc. ECOC, Rome, Italy, September 2018, PDP Th3F.7, DOI: 10.1109/ECOC.2018.8535174

[6] S. Jia, M.-C. Lo, L. Zhang, et al.: 'Integrated Dual-DFB Laser for $408 \mathrm{GHz}$ Carrier Generation Enabling 131 Gbit/s Wireless Transmission over 10.7 Meters', in Proc. OFC, SanDiego, USA, March 2019, paper Th1C.2, DOI: https://doi.org/10.1364/OFC.2019.Th1C.2
[7] X. Pang, S. Jia, O. Ozolins, et al.: 'Single Channel 106 Gbit/s 16QAM Wireless Transmission in the $0.4 \mathrm{THz}$ Band', in Proc. OFC, Los Angeles, USA, March 2017, paper Tu3B.5

[8] M. Freire Hermelo, P.-T. Shih, M. Steeg, et al.: 'Spectral Efficient 64-QAM-OFDM Terahertz Communication Link', Optics Express, 2017, 25, (16), pp. 19360-19370, DOI: https://doi.org/10.1364/OE.25.019360

[9] X. Li, J. Yu, L. Zhao, et al.: '132-Gb/s Photonics-Aided Single-Carrier Wireless Terahertz-Wave Signal Transmission at 450GHz Enabled by 64QAM Modulation and Probabilistic Shaping', in Proc. OFC, SanDiego, USA, March 2019, paper M4F.4, DOI: https://doi.org/10.1364/OFC.2019.M4F.4

[10] L. Zhang, X. Hong, X. Pang, et al.: 'Nonlinearity-Aware 200-Gbit/s Discrete Multi-Tone Transmission for C-band Short-Reach Optical Interconnects with a Single Packaged Electro-Absorption Modulated Laser', Optics Letters, 2018, 43, (2), pp. 182-185, DOI: https://doi.org/10.1364/OL.43.000182

[11] T. Ishibashi, Y. Muramoto, T. Yoshimatsu, et al.: 'UniTraveling-Carrier Photodiodes for Terahertz Applications', IEEE Journal of Selected Topics in Quantum Electronics, 2014, 20, (6), pp. 79-88, DOI: 10.1109/JSTQE.2014.2336537 